\title{
$\sqrt{ }$ |
}

\section{STEM}

\section{Assessing the Role of Fic (Filamentation Induced by cAMP) Proteins in E. coli}

Student researchers: Ashlynn Kokaska, Junior

Fic proteins are evolutionarily conserved from bacteria to humans and are characterized by an amino acid motif of $\mathrm{HxFx}[\mathrm{D} / \mathrm{E} / \mathrm{N}][\mathrm{G} / \mathrm{A}] \mathrm{N}[\mathrm{G} / \mathrm{K}] \mathrm{RxxR}$. Several bacterial Fic proteins act as virulence factors or toxins that post-translationally modify host proteins leading to cell death, a mechanism pathogenic bacteria exploit to evade immune defenses. Fic proteins are inter- or intramolecularly regulated by an inhibitory helix defined by a $(\mathrm{S} / \mathrm{T}) \operatorname{XXXE}(\mathrm{G} / \mathrm{N})$ motif. Therefore, these proteins have been classified as toxin/antitoxin modules. Fic proteins were originally identified in Escherichia coli, where a mutation in the gene encoding the E. coli Fic protein (EcFic) resulted in aberrant cell division. Consequently, E. coli developed a filamentation phenotype in response to heat shock and cAMP, thus the name fic (filamentation induced by cAMP) was coined. Despite the advances made in understanding Fic proteins from pathogenic bacteria, researchers lack a basic understanding of the role of Fic proteins during the life cycle of nonpathogenic bacteria such as E. coli. Our goal is to understand the role of EcFic in regulating cell division in E. coli and why its aberrant function causes filamentation. To this end, we are studying the $E$. coli toxin/antitoxin complex composed of EcFic and its putative inhibitor, YhfG. By co-expressing affinity-tagged versions of EcFic and $\mathrm{YhfG}$, our goal is to identify protein targets with which the EcFic-YhfG complex interacts. These protein targets will be identified by mass spectrometry and then validated and assessed for Fic-mediated modifications. We predict that some of these interacting proteins may be involved in the cell division pathway.

Research advisor Seema Mattoo writes, "Fic proteins regulate critical processes such as bacterial pathogenesis, stress survival, and cancer progression. Ashlynn's work on the founding member of this protein family, Fic from Escherichia coli (EcFic), will identify a new role for Fic proteins in regulating basic cellular processes such as bacterial cell division."

Kokaska, A. (2015). Assessing the role of Fic (filamentation induced by cAMP) proteins in E. coli. Journal of Purdue Undergraduate Research, 5, 90-91. http://dx.doi.org/10.5703/1288284315667 